\title{
Incorporation of Sugar into Conducting Polymer Enables Specific Biosensing of Human Influenza Virus
}

\author{
Tatsuro Goda ${ }^{1}$, Wenfeng Hai ${ }^{1}$, Hiroaki Takeuchi', Shoji Yamaoka ${ }^{1}$, Yukichi Horiguchi', \\ Akira Matsumoto1, Yuji Miyahara ${ }^{1}$, \\ ${ }^{1}$ Tokyo Medical and Dental University (TMDU), \\ 2-3-10 Kanda-Surugadai, Chiyoda, Tokyo 101-0062, Japan \\ goda.bsr@tmd.ac.jp
}

\begin{abstract}
Early detection of influenza virus is key to the prevention of pandemics, which cause up to 500-thousands deaths worldwide each year. Current diagnostic methods are limited by time, sensitivity, cost, or ease of use. We exploited the ability of conjugated polymers to chemically receive a "graft" of 2,6-sialyllactose, a trisaccharide that could recognize and specifically bind to human influenza virus, into their building blocks. Conducting polymers conduct electricity, and their advantages of low toxicity and flexibility make them suitable for use in biological systems as biosensors. This binding was detected by measuring small electrical changes of the functionalized poly(ethylenedioxythiophene) (PEDOT) either by potentiometry or amperometry, enabling the virus to be identified as low as 0.01 hemagglutinating unit (HAU). The potential mass productivity of this approach could allow on-site viral detection, which would be particularly beneficial in remote areas.
\end{abstract}

Key words: PEDOT; Human Influenza Virus; Electropolymerization; Organic Electrochemical Transistors; Potentiometry

\section{Introduction}

Seasonal influenza virus infects $5-15 \%$ of the world's population. A conventional diagnosis method at clinics is an immunochromatographic test (ICT). Because anti-influenza virus therapeutic drugs are effective within 48 hours after onset, early diagnosis is crucial for medication [1]. Therefore, improvements in sensitivity are required for point-of-care testing. Binding of hemagglutinin (HA) to the host cell surface is mediated by trisaccharide receptors on the cell membrane [2]. HA as an antigenic epitope on the virus envelope specifically recognizes the linkage between sialic acid and lactose residues in the trisaccharide. Human influenza viruses preferentially binds to sialyllactose via an $\alpha 2,6^{\prime}$ linkage. Based on this biochemistry, we aimed to develop a 2,6sialyllactose-functionalized conducting polymer. Conducting polymers have features in electrical/ionic conductivities, mechanical flexibility, low cytotoxicity, and processability. We synthesized conjugated copolymers composed of 3,4-ethylenedioxythiophene (EDOT) and its derivative bearing an oxylamine group (EDOTOA) (Fig. 1). EDOTOA reacts with the reducing end of sialyllactose during the bioconjugation reaction. Prompted by the development of wearable sensors for human influenza A virus, we aim to acquire a reliable electrical signal following viral recognition using an organic electrochemical transistor (OECT) as well as potentiometry setups.

\section{Experimental}

EDOT was purchased from Sigma-Aldrich Japan (Tokyo, Japan). EDOTOA was synthesized as previously reported [3]. EDOT and EDOTOA were electro-copolymerized by cyclic voltammetry (CV) from -0.6 to +1.1 to $-0.6 \mathrm{~V}$ for 10 cycles at $0.1 \mathrm{~V} \mathrm{sec}^{-1}$ in water containing $100 \quad \mathrm{mM} \quad \mathrm{NaClO}_{4}$ at room temperature. Electrochemical impedance spectroscopy (EIS) was conducted in 0.01x DPBS ( $\mathrm{pH} 7.4)$ containing $5 \mathrm{mM} \mathrm{Fe}(\mathrm{CN})_{6}{ }^{3-14-}$ at the frequency from $10 \mathrm{kHz}$ to $0.1 \mathrm{~Hz}$ with a 50 $\mathrm{mV}$-AC voltage superimposed on a DC bias of +0.2 V. 2,6-sialyllactose $(100 \mu \mathrm{M}$ in acetic acid, $\mathrm{pH}$ 5.3) was reacted with the EDOTOA unit in $0.01 \times$ Dulbecco's phosphate buffered saline (DPBS) at $60^{\circ} \mathrm{C}$ for $12 \mathrm{~h}$. Human Influenza A Virus (H1N1, PR8 strain) was obtained by cultivation in the chicken embryo and used after fixation in $0.05 \%$ formalin. The binding of human influenza virus on the 2,6-sialyllatosegrafted poly(EDOT-Co-EDOTOA) was determined by quartz crystal microbalance (QCM), OECT, and potentiometry. The binding experiments were performed by injecting virus solution with two times delusion series. 


\section{Results and Discussion}

The oxidization potential was observed at +1.0 $\mathrm{V}$ during electropolymerization. Continuous increases in the cathodic and anodic currents over the CV cycles were observed for 0 and 25 mol\% EDOTOA in feed, indicating a successful electrodeposition onto a electrode surface. By contrast, almost no changes in the currents were observed at 50-100 mol\% EDOTOA. The average step height and arithmetic average of surface roughness of the resultant films decreased by increasing the EDOTOA compositions. Charge transfer resistance increased and constant phase element decreased with an increase in a given EDOTOA composition in EIS. Since the incorporation of EDOTOA showed a trade-off relationship between the electronic/ionic conductivities and target recognition ability, we chose the composition at $25 \mathrm{~mol} \%$ EDOTOA for the subsequent glycosylation and influenza $A$ virus biosensing.

The glycosylation was confirmed by changes in the surface mass and wettability. The introduction rate of sialyllactose to the overall EDOTOA unit in the film was about $52 \mathrm{~mol} \%$.

QCM studies showed preferential binidng of the human influenza virus onto 2,6-sialyllactose- modified PEDOT than 2,3-sialyllactose-modified one at $>0.125 \mathrm{HAU}$. The apparent $\mathrm{K}_{\mathrm{D}}$ and limit of detection (LOD) were was 0.96 and 0.12 $\mathrm{HAU}$, respectively. In potentiometry, the statistically significant signal was obtained at $>0.03 \mathrm{HAU}$ [3]. The apparent $K_{D}$ and LOD were 0.16 and $0.013 \mathrm{HAU}$, respectively. A change in the drain current in OECT was statistically significant at $>0.03 \mathrm{HAU}$ and the LOD was $0.025 \mathrm{HAU}$ [4]. The LOD in potentiometry and OECT is about two orders of magnitude better than that of conventional ICT of $\sim 1 \mathrm{HAU}$.

\section{Conclusions}

We performed sensitive, specific, and label-free biosensing of human influenza $A$ virus using PEDOTs functionalized with 2,6-sialyllactose. EDOTOA was synthesized for the trisaccharide conjugation. Electrochemical copolymerization under optimized conditions resulted in low impedance at the interface. Specific interaction of 2,6-sialyllactose with $\mathrm{HA}$ in the virus envelope allowed label-free sensing by QCM, potentiometry, and OECT. The sensitivity enhanced by two orders of magnitude as compared with commercial kits. Due to the processability and mass productivity, the conducting polymers developed may find applications in wearable monitoring and pointof-care testing of influenza viruses.

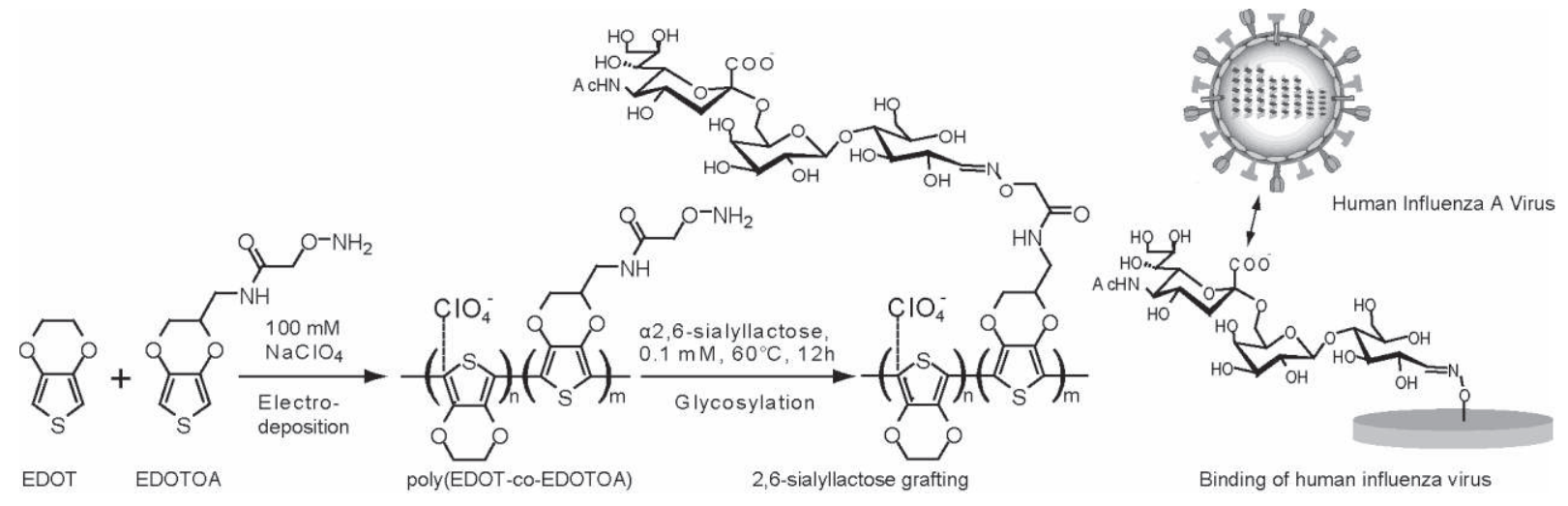

Fig.1. Electrochemical copolymerization of EDOT with EDOTOA in the presence of $\mathrm{NaClO}_{4}$ on a glassy carbon electrode followed by glycosylation of the EDOTOA unit with sialyllactose.

\section{Acknowledgments}

T.G. is grateful for financial support from the Futaba Electronics Foundation, and Asahi Glass Foundation. Part of the research is financially supported by the ImPACT Miyata program from the JST agency.

\section{References}

[1] A. Moscona. Drug therapy - neuraminidase inhibitors for influenza. New. Engl. J. Med. 353, 1363-1373 (2005); doi: 10.1056/NEJMra050740

[2] R.A. Medina, A. Garcia-Sastre. Influenza A viruses: new research developments. Nat. Rev.
Microbiol. 9, 590-603 (2011); doi: 10.1038/nrmicro2613

[3] W. Hai, T. Goda, H. Takeuchi, S. Yamaoka, Y. Horiguchi, A. Matsumoto, Y. Miyahara. Specific recognition of human influenza virus with PEDOT bearing salic acid-terminated trisaccharides. ACS Appl. Mater. Inter. 9, 14162-14170 (2017); doi: 10.1021/acsami.7b02523

[4] W. Hai, T. Goda, H. Takeuchi, S. Yamaoka, Y. Horiguchi, A. Matsumoto, Y. Miyahara. Human Influenza Virus Detection Using SialyllactoseFunctionalized Organic Electrochemical Transistors. Sens.Actuat. B-Chem. 260, 635-641 (2018); doi: 10.1016/j.snb.2018.01.081 